\title{
The effects of element shape on the critical time step in explicit time integrators for elasto-dynamics
}

\author{
Harm Askes ${ }^{1, * \dagger}$, Antonio Rodríguez-Ferran $^{2}$ and Jack Hetherington ${ }^{1}$ \\ ${ }^{1}$ Department of Civil and Structural Engineering, University of Sheffield, Sheffield, United Kingdom \\ ${ }^{2}$ Laboratori de Càlcul Numèric, Universitat Politècnica de Catalunya, Barcelona, Spain
}

\begin{abstract}
SUMMARY
In this paper, the effects of element shape on the critical time step are investigated. The common ruleof-thumb, used in practice, is that the critical time step is set by the shortest distance within an element divided by the dilatational (compressive) wave speed, with a modest safety factor. For regularly shaped elements, many analytical solutions for the critical time step are available, but this paper focusses on distorted element shapes. The main purpose is to verify whether element distortion adversely affects the critical time step or not. Two types of element distortion will be considered, namely aspect ratio distortion and angular distortion, and two particular elements will be studied: four-noded bilinear quadrilaterals and three-noded linear triangles. The maximum eigenfrequencies of the distorted elements are determined and compared to those of the corresponding undistorted elements. The critical time steps obtained from single element calculations are also compared to those from calculations based on finite element patches with multiple elements. Copyright (C) 2014 John Wiley \& Sons, Ltd.
\end{abstract}

KEY WORDS: explicit time integration; critical time step; eigenfrequency; element distortion; element aspect ratio

\section{INTRODUCTION}

Explicit time integration methods are the most popular methods to solve the dynamic equations of fast-transient processes. Combined with lumped mass matrices, explicit algorithms require minimal CPU time and computer memory per time step, and they are very simple to implement. However, a significant drawback is that explicit time integrators are generally only conditionally stable. That is, the time step used for time integration must be chosen smaller than the so-called critical time step for the simulation to remain stable.

Much research has been carried out to compute, and subsequently control, the critical time step. For low-order elements with regular shapes, it may be possible to derive the critical time step in a closed-form expression, see for instance [1]. However, in more general applications, the critical time step has to be estimated, and it is important for practitioners that reliable rules-of-thumb are provided that are safe but as close to the exact (yet potentially unknown) values of the critical time step as possible. In what follows, the focus will be on the elasto-dynamics of solids and structures for which the spatial discretisation is performed using the finite element method.

\footnotetext{
*Correspondence to: Harm Askes, Department of Civil and Structural Engineering, University of Sheffield, Mappin Street, Sheffield S1 3JD, United Kingdom.

†E-mail: h.askes@sheffield.ac.uk
} 


\subsection{Computing the critical time step}

For explicit time integration schemes, such as the central difference scheme, the critical time step $\Delta t_{\text {crit }}$ is given by Hughes [2]

$$
\Delta t_{\text {crit }}=\frac{2}{\omega_{\max }^{G}}
$$

where $\omega_{\max }^{G}$ is the maximum eigenfrequency of the entire finite element assembly (the superscript $G$ indicates that this is a global quantity). In order to find the maximum eigenfrequency, a global eigenvalue problem must thus be solved. This is usually deemed to be too expensive, and most researchers solve instead the maximum eigenfrequency of a single element. The maximum eigenvalue of the entire finite element mesh is smaller than the maximum eigenvalue of the individual elements of that mesh [3]; therefore, it is safe to estimate the critical time step using the maximum eigenfrequency of the smallest finite element.

The eigenvalue problem of a single element is a polynomial equation, whereby the degree of the polynomial equals the number of degrees of freedom of that particular element. Generally, analytical solutions can only be guaranteed for polynomial equations up to degree four (i.e., quartic equations), which would restrict analytical solutions of element eigenvalue problems to finite elements that have at most four degrees of freedom (e.g. two-noded bar elements and two-noded beam elements). However, the eigenfrequencies corresponding to rigid-body motions are zero, and the resulting polynomial equations can usually be simplified considerably. Moreover, additional assumptions such as reduced numerical integration and focus on the dilatational deformation mode may lead to further simplifications. Thus, eigenvalues have been obtained for quadrilateral and hexahedral elements [4] and for Mindlin plate elements [5]. More recently, the use of symbolic operation software has enabled the evaluation of eigenvalue problems for more complicated finite elements, such as fournoded and eight-noded quadrilaterals using lumped or consistent mass matrices and reduced or full integration schemes [1].

In the literature, there are two interesting exceptions to the general observation that most researchers focus on the element eigenvalue problem. Firstly, Lin [3,6] suggested to use the eigenvalue problem at the integration point level, rather than the element level. The presented bounds on the respective eigenvalues demonstrate that this approach is safe but conservative: the critical time step computed using the integration point eigenvalues is around $15 \%$ lower than the critical time step computed using the elemental eigenvalues for the tests reported in [3] and around 30\% lower than the global maximum eigenvalue for the tests reported in [6]. Alternatively, some researchers aim to solve the global eigenvalue problem, using for instance power iteration methods [7] or Lanczos methods [8]. If the difference between global maximum eigenfrequency and elemental maximum frequency is large, such methods may lead to a significant increase in time step size. Although this could lead to reduced CPU times, this is off-set by the increased computational effort required to solve the global eigenvalue problem, which can be considerable. Furthermore, such global methods may even be unsafe in that they over-estimate the critical time step-a problem that has been mentioned, and solved, in [8].

\subsection{Controlling the critical time step}

It is generally found that the relevant factors that determine the critical time step are the wave speed of the material (in particular the dilatational one), the nature of the higher-order eigenmodes of the finite element, and the element geometry. This knowledge has been used in the literature to control, and indeed manipulate, the critical time step.

The wave speed can be decreased (thereby increasing the critical time step) by increasing the mass density or decreasing the stiffness of the material. Obviously, these rather crude methods change the mechanical properties of the system, but they have been successfully applied to the simulation of relatively slow processes. For instance, increasing the mass density (often denoted as 'mass scaling') has been reported to be satisfactory in metal forming simulations [9-13], whereas decreasing the stiffness has been suggested for the simulation of surgery applications [14]. However, for faster 
processes, such unphysical modifications are usually not recommendable [15]. Locally, the wave speed may also be affected by penalty functions used to impose constraints. As such, the critical time step can be decreased by the use of stiffness-type penalties or increased via mass-type penalties [16]; using both types of penalty at the same time can then be used to keep the critical time step unmodified [17-19].

An important notion is that the critical time step is set by the largest eigenvalue; therefore, physical properties may be adjusted such that the larger eigenvalues are lowered, whereas the smaller eigenvalues remain unaffected. This has been the philosophy of more sophisticated adjustments to the mass matrix such as those suggested in [15, 20-22]. In these applications, a non-diagonal matrix is added to the mass matrix such that the total mass is conserved, but the larger eigenvalues are lowered-often significantly. Whilst at first sight this may seem to be yet another unrealistic and unphysical way of adjusting the material properties, it was shown recently that such approaches are in fact equivalent to introducing micro-structural inertia in the material properties in the spirit of gradient-enriched continuum theories [23]. However, the fact that this approach relies on nondiagonal mass matrices is a significant drawback. To address this, it has been suggested to use iterative solution methods [24], to use the micro-inertia effects only in those parts of the domain where the element sizes are small [23], or to modify the micro-inertial mass matrix according to a Neumann expansion in order to preserve its diagonal structure [25].

\subsection{Effects of element shape}

An aspect that has remained relatively under-emphasized in the literature is the element shape. Whilst it is well-known that the critical time step is proportional to the element size (usually taken to be the smallest distance between any two non-adjacent edges or faces of the element), the effects of non-regular element shapes seem to have received far less attention in the literature-a notable exception for heat problems is due to Myers [26]. This is indeed the focus of the present paper.

The apparent stiffness of a finite element assembly may be increased by element distortion. Thus, there is an intuitive argument that such an artificially increased stiffness may lead to artificially increased wave speeds and, in turn, artificially decreased critical time steps. If this turns out to be true, guidance to practitioners must be provided regarding the extend to which the critical time step is affected, so that this can be accounted for.

In Sections 4-7, the effects will be studied of aspect ratio distortion and angular distortion on four-noded quadrilateral and three-noded triangular elements. In Section 8, the maximum eigenfrequencies of distorted elements are then compared to the maximum eigenfrequencies of undistorted elements. Next, assemblies of multiple finite elements are studied in Section 9. First, however, some relevant fundamentals and the eigenfrequencies of undistorted elements are revisited in Sections 2 and 3, respectively. Throughout, use has been made of the symbolic operation software Maple for the single element computations, whereas MATLAB has been used for the computations with multiple elements in Section 9.

\section{METHODOLOGY}

The maximum eigenfrequency required in Equation (1) is approximated by selecting the largest root $\omega$ from the dynamic eigenvalue problem of a single element, that is

$$
\operatorname{det}\left(-\omega^{2} \mathbf{M}+\mathbf{K}\right)=0
$$

where $\mathbf{M}$ and $\mathbf{K}$ are the element mass matrix and the element stiffness matrix, respectively.

In explicit dynamics, a lumped mass matrix is commonly used, the diagonal entries of which are obtained from the consistent mass matrix by applying the row-sum technique, for instance. For the stiffness matrix, different numerical integration schemes may be used, such as full integration (the default option) or selective reduced integration (applying under-integration for the shear deformation terms but full integration for the normal deformation terms); in this paper, the main focus is on full integration, but characteristic polynomials for reduced integration and selective reduced integration are given in Appendices A and B, respectively. Ling and Cherukuri provide a comprehensive 
review of different square element types and different integration rules, whilst they also cover the plane stress option alongside the plane strain assumption [1].

It is convenient to express the maximum eigenfrequency and, thus, the critical time step in terms of the wave speed of the material. In two-dimensional configurations, the dilatational wave speed $c_{d}$ and the shear wave speed $c_{s}$ are given by

$$
\begin{gathered}
c_{d}=\sqrt{\frac{\lambda+2 \mu}{\rho}} \\
c_{s}=\sqrt{\frac{\mu}{\rho}}
\end{gathered}
$$

where $\lambda$ and $\mu$ are the usual Lamé constants and $\rho$ is the mass density of the material. It is also useful to define the ratio $q$ between shear and dilatational wave speed [1], which is a function of Poisson's ratio $v$ only:

$$
q=\frac{c_{s}}{c_{d}}=\sqrt{\frac{1-2 v}{2-2 v}}
$$

For linear elastic, isotropic materials, Poisson's ratio $-1 \leqslant v \leqslant \frac{1}{2}$. Therefore, for such materials, the dilatational wave speed is larger than the shear wave speed. As a result, the critical time step is dictated by the dilatational wave speed, which is confirmed when checking the eigenmodes that correspond to the maximum eigenfrequency.

The aforementioned observations have helped many users of finite element packages to find simple estimates for the critical time step. An intuitive interpretation of the critical time step is that waves should not be allowed to travel too quickly through an element. Thus, the critical time step is linked to the speed of the dilatational waves and the shortest travel distance within an element. With this in mind, the objective of this paper can be formulated as follows: verify whether element distortion is adversal or beneficial to the rule-of-thumb that the critical time step is set by the shortest distance divided by the dilatational wave speed. To do this in a systematic manner, the effects of element distortion will be studied whilst keeping the wave speed and the shortest distance constant. Here, 'shortest distance' is defined as the shortest distance between any node and a non-adjacent edge.

\section{EIGENFREQUENCIES OF UNDISTORTED ELEMENTS}

In order to provide reference cases for the derivations later in this paper, first, the eigenfrequencies for a number of undistorted elements are computed. In two spatial dimensions, the element shape function matrix $\mathbf{N}$ collects the finite element shape functions $\phi_{i}$ through

$$
\mathbf{N}=\left[\begin{array}{ccccc}
\phi_{1} & 0 & \phi_{2} & 0 & \ldots \\
0 & \phi_{1} & 0 & \phi_{2} & \ldots
\end{array}\right]
$$

The element consistent mass matrix $\mathbf{M}^{\text {cons }}$ is obtained from

$$
\mathbf{M}^{\text {cons }}=\int_{\Omega_{\mathrm{el}}} \mathbf{N}^{T} \rho \mathbf{N} \mathrm{d} V
$$

and can subsequently be used to find the element lumped mass matrix $\mathbf{M}^{\text {lump }}$ via

$$
M_{i i}^{\text {lump }}=\sum_{j} M_{i j}^{\text {cons }}
$$


The element stiffness matrix $\mathbf{K}$ is written as

$$
\mathbf{K}=\int_{\Omega_{\mathrm{el}}} \mathbf{B}^{T} \mathbf{D B} \mathrm{d} V
$$

where

$$
\mathbf{B}=\left[\begin{array}{ccccc}
\frac{\partial \phi_{1}}{\partial x} & 0 & \frac{\partial \phi_{2}}{\partial x} & 0 & \ldots \\
0 & \frac{\partial \phi_{1}}{\partial y} & 0 & \frac{\partial \phi_{2}}{\partial y} & \cdots \\
\frac{\partial \phi_{1}}{\partial y} & \frac{\partial \phi_{1}}{\partial x} & \frac{\partial \phi_{2}}{\partial y} & \frac{\partial \phi_{2}}{\partial x} & \cdots
\end{array}\right]
$$

The material stiffness matrix $\mathbf{D}$ for plane strain is given in terms of the mass density $\rho$ and the wave speeds $c_{d}$ and $c_{s}$ from Equation (3) as

$$
\mathbf{D}=\rho \cdot\left[\begin{array}{ccc}
c_{d}^{2} & c_{d}^{2}-2 c_{s}^{2} & 0 \\
c_{d}^{2}-2 c_{s}^{2} & c_{d}^{2} & 0 \\
0 & 0 & c_{s}^{2}
\end{array}\right]
$$

The element lumped mass matrix $\mathbf{M}^{\text {lump }}$ and the element stiffness matrix $\mathbf{K}$ are substituted into Equation (2), after which this expression is solved for the eigenfrequencies $\boldsymbol{\omega}$. In order to compute the critical time step, the largest eigenfrequency must be selected and substituted into Equation (1). Typically, the value of Poisson's ratio $v$ determines which of the eigenfrequencies is the largest.

\subsection{Four-noded square element}

For square elements with edge length $h$ and using full integration, Equation (2) can be expanded as

$$
\omega^{6} h^{6}\left(3 \omega^{2} h^{2}-4 c_{d}^{2}-4 c_{s}^{2}\right)^{2}\left(\omega^{2} h^{2}-8 c_{s}^{2}\right)^{2}\left(\omega^{2} h^{2}-8 c_{d}^{2}+8 c_{s}^{2}\right)=0
$$

The (square of the) eigenfrequencies are thus found, using Equation (4), as

$$
\begin{aligned}
& \omega_{1}^{2}=\omega_{2}^{2}=\omega_{3}^{2}=0 \\
& \omega_{4}^{2}=\omega_{5}^{2}=\frac{4 c_{d}^{2}\left(1+q^{2}\right)}{3 h^{2}} \\
& \omega_{6}^{2}=\omega_{7}^{2}=\frac{8 c_{d}^{2} q^{2}}{h^{2}} \\
& \omega_{8}^{2}=\frac{8 c_{d}^{2}\left(1-q^{2}\right)}{h^{2}}
\end{aligned}
$$

The zero eigenfrequencies in Equation (12a) correspond to the rigid body motions. The eigenfrequencies (normalised with respect to $h / c_{d}$ ) are plotted against Poisson's ratio in Figure 1 (left). It can be seen that the largest eigenfrequency is $\omega_{8}$ for positive values of $v$ and $\omega_{6}=\omega_{7}$ for negative values of $v$. Note that $\omega_{\max }=2 c_{d} / h$ for $v=0$ which, upon substitution into Equation (1), leads to the well-known rule-of-thumb that $\Delta t_{\text {crit }}=$ 'element size' / wave speed', although this rule-of-thumb must be applied with some safety factor for other values of Poisson's ratio. 

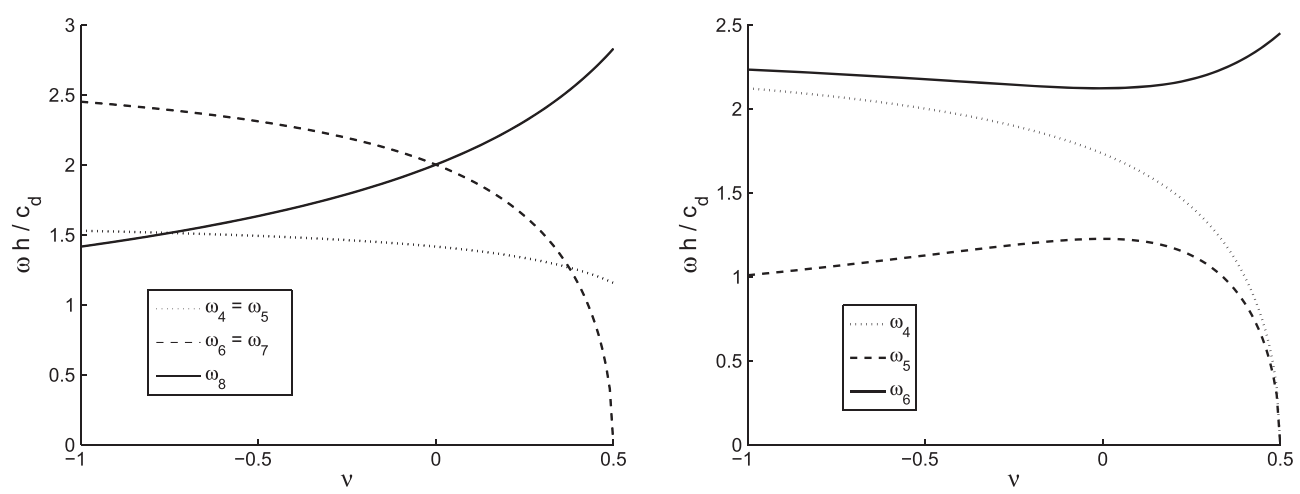

Figure 1. Normalised eigenfrequencies $\omega h / c_{d}$ against Poisson's ratio $v$ for square elements (left) and for right-angled isosceles elements (right).

\subsection{Three-noded equilateral triangle}

The shape functions for a three-noded equilaterial triangle with height $h$ can be written as

$$
\begin{aligned}
\phi_{1} & =\frac{h-x \sqrt{3}-y}{2 h} \\
\phi_{2} & =\frac{h+x \sqrt{3}-y}{2 h} \\
\phi_{3} & =\frac{y}{h}
\end{aligned}
$$

where the origin of the Cartesian coordinate system has been chosen halfway the base of the triangle. The eigenvalue problem of Equation (2) then becomes

$$
\omega^{6} h^{6}\left(\omega^{2} h^{2}-9 c_{s}^{2}\right)^{2}\left(\omega^{2} h^{2}-9 c_{d}^{2}+9 c_{s}^{2}\right)=0
$$

which yields

$$
\begin{aligned}
& \omega_{1}^{2}=\omega_{2}^{2}=\omega_{3}^{2}=0 \\
& \omega_{4}^{2}=\omega_{5}^{2}=\frac{9 c_{d}^{2} q^{2}}{h^{2}} \\
& \omega_{6}^{2}=\frac{9 c_{d}^{2}\left(1-q^{2}\right)}{h^{2}}
\end{aligned}
$$

Similar to the square element discussed in the preceding text, it can be easily verified that the largest eigenfrequency is $\omega_{4}=\omega_{5}$ for negative Poisson's ratios and $\omega_{6}$ for positive Poisson's ratios.

\subsection{Three-noded right-angled isosceles triangle}

Although the equilateral triangle is probably the most regularly shaped triangle from a purely geometric point of view, it is also worthwhile to document the eigenfrequencies of a right-angled isosceles triangle because such elements would appear in structured meshes for rectangular domains. Taking the length of the hypothenuse to be equal to $2 h$ so that the height of the triangle is again $h$, the shape functions can be written as 


$$
\begin{aligned}
\phi_{1} & =\frac{h-x-y}{2 h} \\
\phi_{2} & =\frac{h+x-y}{2 h} \\
\phi_{3} & =\frac{y}{h}
\end{aligned}
$$

The eigenvalue equation problem reads

$$
\omega^{6} h^{6}\left(\omega^{2} h^{2}-6 c_{s}^{2}\right)\left(\omega^{4} h^{4}-6 \omega^{2} h^{2} c_{d}^{2}+27 c_{d}^{2} c_{s}^{2}-27 c_{s}^{4}\right)=0
$$

so that

$$
\begin{aligned}
& \omega_{1}^{2}=\omega_{2}^{2}=\omega_{3}^{2}=0 \\
& \omega_{4}^{2}=\frac{6 c_{d}^{2} q^{2}}{h^{2}} \\
& \omega_{5}^{2}=\frac{3 c_{d}^{2}}{h^{2}}\left(1-\sqrt{1-3 q^{2}\left(1-q^{2}\right)}\right) \\
& \omega_{6}^{2}=\frac{3 c_{d}^{2}}{h^{2}}\left(1+\sqrt{1-3 q^{2}\left(1-q^{2}\right)}\right)
\end{aligned}
$$

The non-zero eigenfrequencies of Equation (18) are plotted against Poisson's ratio in Figure 1 (right), and it can be seen that $\omega_{6}$ is the largest eigenfrequency for all values of $v$.

\section{THE EFFECTS OF ASPECT RATIO DISTORTION ON RECTANGULAR ELEMENTS}

The first type of element distortion that will be considered is denoted as 'aspect ratio distortion' of rectangular elements. Applying aspect ratio distortion to a square, the dimensions of the (now rectangular) element can be indicated with $h$ and $\alpha \cdot h$, respectively—see Figure 2. Thus, $\alpha>1$ sets the magnitude of aspect ratio distortion whilst maintaining $h$ as the notation for the shortest distance within the element.

Including the aspect ratio parameter $\alpha$ into the evaluation of Equation (2) results in a polynomial equation for $\omega$ as

$$
\begin{aligned}
& \omega^{6} h^{6}\left(3 \omega^{2} h^{2} \alpha^{2}-4 \alpha^{2} c_{d}^{2}-4 c_{s}^{2}\right)\left(3 \omega^{2} h^{2} \alpha^{2}-4 c_{d}^{2}-4 \alpha^{2} c_{s}^{2}\right)\left(\omega^{2} h^{2} \alpha^{2}-4 \alpha^{2} c_{s}^{2}-4 c_{s}^{2}\right) \\
& \times\left(\omega^{4} h^{4} \alpha^{2}-4 \omega^{2} h^{2}\left(\alpha^{2}+1\right) c_{d}^{2}+64 c_{d}^{2} c_{s}^{2}-64 c_{s}^{4}\right)=0 \\
& 19 \begin{array}{r}
-\ldots \\
1 \\
1 \\
-\ldots
\end{array}
\end{aligned}
$$

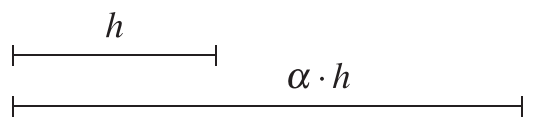

Figure 2. Aspect ratio distortion for rectangular elements. 
which yields

$$
\begin{aligned}
& \omega_{1}^{2}=\omega_{2}^{2}=\omega_{3}^{2}=0 \\
& \omega_{4}^{2}=\frac{4 c_{d}^{2}\left(\alpha^{2}+q^{2}\right)}{3 \alpha^{2} h^{2}} \\
& \omega_{5}^{2}=\frac{4 c_{d}^{2}\left(\alpha^{2} q^{2}+1\right)}{3 \alpha^{2} h^{2}} \\
& \omega_{6}^{2}=\frac{4 c_{d}^{2} q^{2}\left(\alpha^{2}+1\right)}{\alpha^{2} h^{2}} \\
& \omega_{7}^{2}=\frac{2 c_{d}^{2}}{\alpha^{2} h^{2}}\left(\alpha^{2}+1-\sqrt{\left(\alpha^{2}+1\right)^{2}-16 \alpha^{2} q^{2}\left(1-q^{2}\right)}\right) \\
& \omega_{8}^{2}=\frac{2 c_{d}^{2}}{\alpha^{2} h^{2}}\left(\alpha^{2}+1+\sqrt{\left(\alpha^{2}+1\right)^{2}-16 \alpha^{2} q^{2}\left(1-q^{2}\right)}\right)
\end{aligned}
$$

In Figure 3, the non-zero eigenfrequencies $\omega_{4}-\omega_{7}$ are normalised with respect to $\omega_{8}$ and plotted for a range of aspect ratios $\alpha$ and a range of Poisson's ratios $v$. Because these ratios of eigenfrequencies are never larger than one, it can be concluded that $\omega_{8}$ as given in Equation (20f) is the maximum eigenfrequency for a rectangular element with full integration.

\section{THE EFFECTS OF ASPECT RATIO DISTORTION ON RIGHT-ANGLED TRIANGLES}

Next, aspect ratio distortion is applied to right-angled triangles. The shortest distance within the triangle is the altitude from the right angle towards the hypothenuse, and this distance is kept constant at $h$. Several parametrisations are possible to capture this aspect ratio distortion, but the one that leads to the simplest expressions is that based on the angle $\theta$ depicted in Figure 4. The coordinates
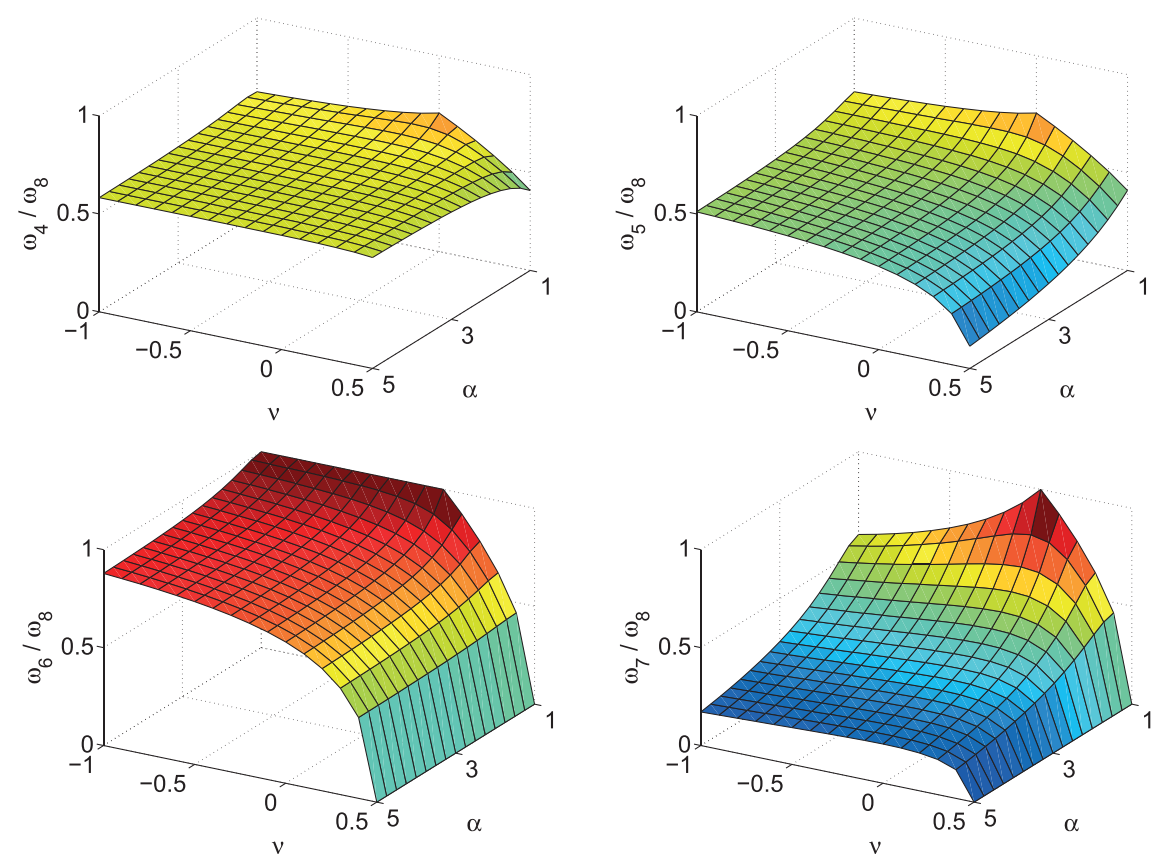

Figure 3. Rectangular elements: ratio of eigenfrequencies $\omega_{4} / \omega_{8}$ (top left), $\omega_{5} / \omega_{8}$ (top right), $\omega_{6} / \omega_{8}$ (bottom left), and $\omega_{7} / \omega_{8}$ (bottom right). 
of the triangle can then be quantified by the dimensions $a=h / \sin (\theta)$ and $b=h / \cos (\theta)$, and the associated eigenvalue problem leads to the following polynomial equation:

$$
\omega^{6} h^{6}\left(\omega^{2} h^{2}-6 c_{s}^{2}\right)\left(\omega^{4} h^{4}-6 \omega^{2} h^{2} c_{d}^{2}+27 \sin ^{2}(2 \theta)\left(c_{d}^{2}-c_{s}^{2}\right) c_{s}^{2}\right)=0
$$

Therefore,

$$
\begin{aligned}
& \omega_{1}^{2}=\omega_{2}^{2}=\omega_{3}^{2}=0 \\
& \omega_{4}^{2}=\frac{6 c_{d}^{2} q^{2}}{h^{2}} \\
& \omega_{5}^{2}=\frac{3 c_{d}^{2}}{h^{2}}\left(1-\sqrt{1-3 \sin ^{2}(2 \theta) q^{2}\left(1-q^{2}\right)}\right) \\
& \omega_{6}^{2}=\frac{3 c_{d}^{2}}{h^{2}}\left(1+\sqrt{1-3 \sin ^{2}(2 \theta) q^{2}\left(1-q^{2}\right)}\right)
\end{aligned}
$$

Because $0 \leqslant q^{2} \leqslant \frac{3}{4}$, the maximum value that $\omega_{4}^{2}$ can attain is $\omega_{4}^{2}=9 c_{d}^{2} / 2 h^{2}$. Furthermore, $0 \leqslant q^{2}\left(1-q^{2}\right) \leqslant \frac{1}{4}$, so that the minimum value that $\omega_{6}^{2}$ can attain is $\omega_{6}^{2}=9 c_{d}^{2} / 2 h^{2}$. Therefore, it can be concluded that $\omega_{4} \ngtr \omega_{6}$. It is also clear that $\omega_{5} \leqslant \omega_{6}$; thus, $\omega_{6}$ is the largest eigenfrequency for a right-angled three-noded triangle.

\section{THE EFFECTS OF ANGULAR DISTORTION ON RHOMBIC ELEMENTS}

Next, angular distortion is studied for square elements turning into rhombic-shaped elements. The main parameter of distortion is the change of the internal angles of the element, indicated with $\theta$ as in Figure 5. If the shortest distance of the element is to be kept constant at $h$, each edge of the element has length $h / \cos (\theta)$. It is possible to express the shape functions directly in Cartesian coordinates, but this leads to lengthy and cumbersome derivations. More transparent expressions are obtained if isoparametric shape functions are formulated in the usual local coordinates $\xi$ and $\eta$, which for rhombic shapes are related to the Cartesian coordinates $x$ and $y$ via $x=\xi / \cos (\theta)+\eta \tan (\theta)$ and $y=\eta$. The Jacobian matrix relating global to local coordinates only depends on the angle $\theta$ and is thus constant throughout the element.

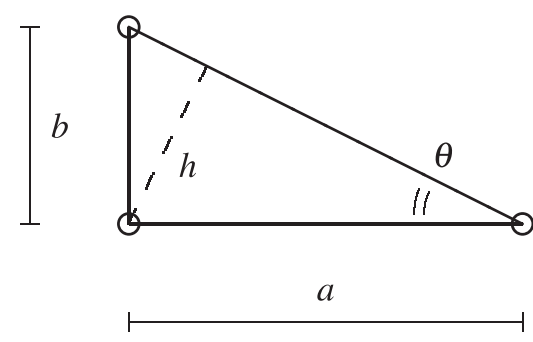

Figure 4. Aspect ratio distortion for right-angled triangular elements.

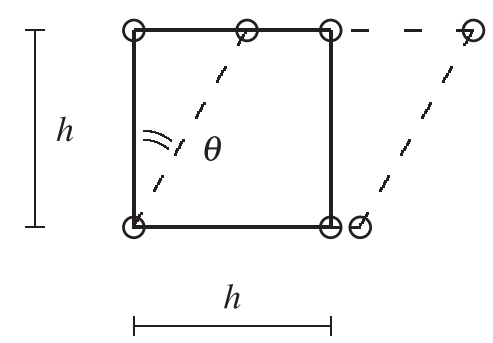

Figure 5. Angular distortion for rhombic elements. 
With these preliminaries, the eigenvalue problem of Equation (2) reduces to

$$
\begin{aligned}
& \omega^{6} h^{6}\left(9 \omega^{4} h^{4}-24 \omega^{2} h^{2}\left(c_{d}^{2}+c_{s}^{2}\right)+16 \cos ^{2}(\theta)\left(c_{d}^{2}-c_{s}^{2}\right)^{2}+64 c_{d}^{2} c_{s}^{2}\right) \\
& \times\left(\omega^{2} h^{2}-8 c_{s}^{2}\right)\left(\omega^{4} h^{4}-8 \omega^{2} h^{2} c_{d}^{2}+64 \cos ^{2}(\theta) c_{d}^{2} c_{s}^{2}-64 \cos ^{2}(\theta) c_{s}^{4}\right)=0
\end{aligned}
$$

Accordingly, the eigenfrequencies of the element are given by

$$
\begin{aligned}
& \omega_{1}^{2}=\omega_{2}^{2}=\omega_{3}^{2}=0 \\
& \omega_{4}^{2}=\frac{4 c_{d}^{2}}{3 h^{2}}\left(1+q^{2}-\left(1-q^{2}\right) \sin (\theta)\right) \\
& \omega_{5}^{2}=\frac{4 c_{d}^{2}}{3 h^{2}}\left(1+q^{2}+\left(1-q^{2}\right) \sin (\theta)\right) \\
& \omega_{6}^{2}=\frac{8 c_{d}^{2} q^{2}}{h^{2}} \\
& \omega_{7}^{2}=\frac{4 c_{d}^{2}}{h^{2}}\left(1-\sqrt{1-4 \cos ^{2}(\theta) q^{2}\left(1-q^{2}\right)}\right) \\
& \omega_{8}^{2}=\frac{4 c_{d}^{2}}{h^{2}}\left(1+\sqrt{1-4 \cos ^{2}(\theta) q^{2}\left(1-q^{2}\right)}\right)
\end{aligned}
$$

The eigenfrequencies of Equation (12) are retrieved by taking $\theta=0$.

With $0 \leqslant q^{2} \leqslant \frac{3}{4}$ and taking $0 \leqslant \theta \leqslant 90^{\circ}$, it can be stated that $\omega_{4} \leqslant \omega_{5}$ and $\omega_{7} \leqslant \omega_{8}$. In Figure 6, the two eigenfrequencies $\omega_{5}$ and $\omega_{6}$ are plotted normalised with respect to $\omega_{8}$, which shows that $\omega_{5}<\omega_{8}$ and $\omega_{6} \leqslant \omega_{8}$. Therefore, the largest eigenfrequency is $\omega_{8}$.

\section{THE EFFECTS OF ANGULAR DISTORTION ON ISOSCELES TRIANGULAR ELEMENTS}

Applying angular distortion to isosceles triangles is most conveniently performed by taking the length of the longest side equal to $2 \alpha h$, whereby the distortion parameter $\alpha$ is taken as $\alpha>\frac{1}{3} \sqrt{3}$ in order to keep the shortest distance of the triangle equal to $h$ (Figure 7). Including the angular distortion in the eigenvalue problem of Equation (2) results in

$$
\omega^{6} h^{6}\left(2 \omega^{2} h^{2} \alpha^{2}-3\left(3 \alpha^{2}+1\right) c_{s}^{2}\right)\left(2 \omega^{4} h^{4} \alpha^{2}-3 \omega^{2} h^{2}\left(3 \alpha^{2}+1\right) c_{d}^{2}+54 c_{d}^{2} c_{s}^{2}-54 c_{s}^{4}\right)=0
$$
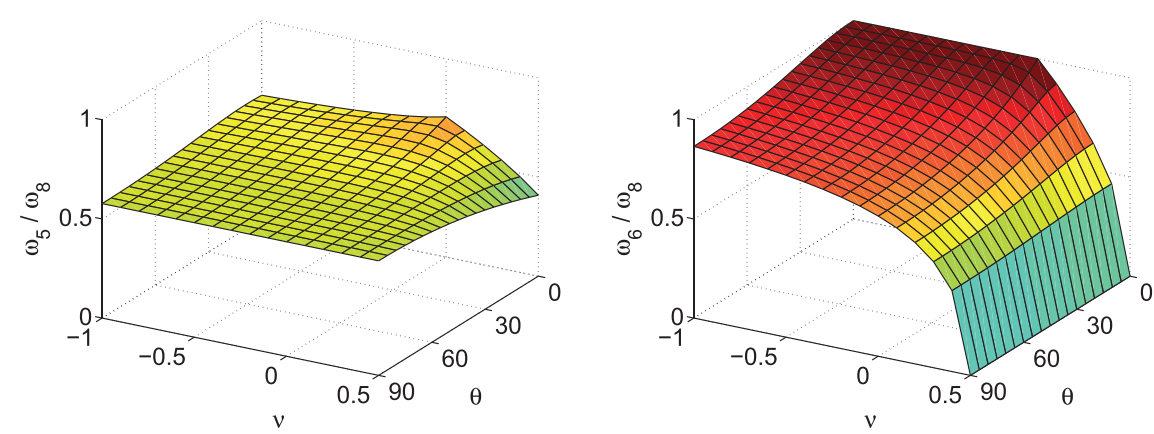

Figure 6. Rhombic elements: ratio of eigenfrequencies $\omega_{5} / \omega_{8}$ (left) and $\omega_{6} / \omega_{8}$ (right). 
which leads to

$$
\begin{aligned}
& \omega_{1}^{2}=\omega_{2}^{2}=\omega_{3}^{2}=0 \\
& \omega_{4}^{2}=\frac{3 c_{d}^{2}\left(3 \alpha^{2}+1\right) q^{2}}{2 \alpha^{2} h^{2}} \\
& \omega_{5}^{2}=\frac{3 c_{d}^{2}}{4 \alpha^{2} h^{2}}\left(3 \alpha^{2}+1-\sqrt{\left(3 \alpha^{2}+1\right)^{2}-48 \alpha^{2} q^{2}\left(1-q^{2}\right)}\right) \\
& \omega_{6}^{2}=\frac{3 c_{d}^{2}}{4 \alpha^{2} h^{2}}\left(3 \alpha^{2}+1+\sqrt{\left(3 \alpha^{2}+1\right)^{2}-48 \alpha^{2} q^{2}\left(1-q^{2}\right)}\right)
\end{aligned}
$$

Figure 8 depicts the ratio of $\omega_{4}$ divided by $\omega_{6}$ for a range of values for the distortion parameter $\alpha$ and the Poisson's ratio $\nu$, and it can be verified that $\omega_{4} \leqslant \omega_{6}$. Furthermore, from Equation (26), it is clear that $\omega_{5} \leqslant \omega_{6}$. Therefore, the largest eigenfrequency for a three-noded isosceles triangle is given by $\omega_{6}$.

\section{COMPARING DISTORTED AND UNDISTORTED ELEMENTS}

Now that the maximum eigenfrequencies of the distorted elements have been determined, it is of interest to compare these to the maximum eigenfrequencies of the associated undistorted elements. Because the shortest distance in all expressions for the eigenfrequencies has been denoted by $h$ throughout, it can be verified whether element distortion has an adverse or beneficial effect on the maximum eigenfrequency.

The quantity of interest is the maximum eigenfrequency of the distorted element divided by the maximum eigenfrequency of the corresponding undistorted element. In particular,

- For aspect ratio distortion of quadrilaterals, $\omega_{8}$ from Equation (20f) is divided by $\omega_{6}$ from Equation (12c) or $\omega_{8}$ from Equation (12d), as appropriate. The result is plotted in Figure 9, top left. It can be verified that the maximum eigenfrequency of a rectangle is never larger than the maximum eigenfrequency of a square with identical shortest distance.
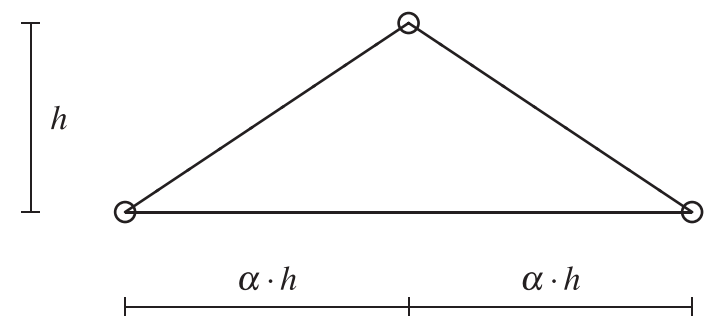

Figure 7. Angular distortion for isosceles triangular elements.

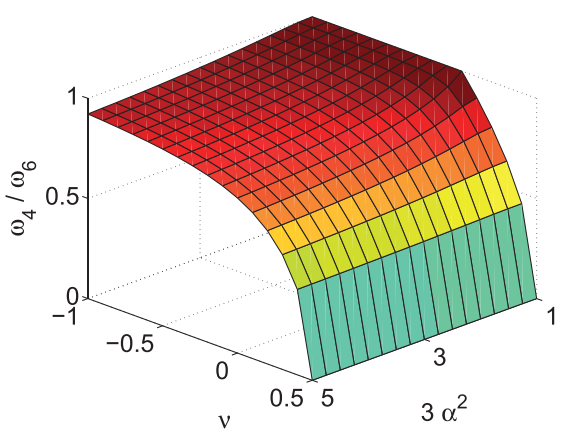

Figure 8. Isosceles triangles, ratio of eigenfrequencies $\omega_{4} / \omega_{6}$. 
- For aspect ratio distortion of right-angled triangles, $\omega_{6}$ from Equation (22d) is divided by $\omega_{6}$ from Equation (18d). The result is plotted in Figure 9, top right. In this case, element distortion does lead to an increase of the maximum eigenfrequency, but this increase is relatively small. The largest increase equals $2 / \sqrt{3} \approx 1.155$, which occurs for the hypothetical combination of Poisson's ratio $v=0$ and distortion angle $\theta=0$. However, if one imposes a bound on the maximum acceptable distortion during mesh generation (that is, an upper bound on the ratio $a / b$, or a lower bound on angle $\theta$ ), the largest increase in the eigenfrequency would be less than $2 / \sqrt{3}$.

- For angular distortion of quadrilaterals, $\omega_{8}$ from Equation (24f) is divided by $\omega_{6}$ from Equation (12c) or $\omega_{8}$ from Equation (12d), as appropriate. The result is plotted in Figure 9, bottom left. Also in this case, element distortion leads to a modest increase in the maximum eigenfrequency. The largest increase equals $\sqrt{2}$ and is obtained for the limit case of degenerate element geometry via distortion angle $\theta=90^{\circ}$ combined with Poisson's ratio $v=0$. Again, this type of distortion can be controlled during mesh generation so that increase factors lower than $\sqrt{2}$ will then be obtained.

- For angular distortion of isosceles triangles, $\omega_{6}$ from Equation (26d) is divided by $\omega_{4}$ from Equation (15b) or $\omega_{6}$ from Equation (15c), as appropriate. The result is plotted in Figure 9, bottom right. As can be seen, the maximum eigenfrequency of a distorted isosceles triangle is never larger than the maximum eigenfrequency of an equilateral triangle with identical shortest distance.

Perhaps the most pertinent conclusion is that the effects of element distortion on the maximum eigenfrequency (and, in turn, the critical time step) are limited: in none of the studied cases was an unbounded increase factor found. Thus, the intuitive notion that the critical time step is set by the shortest distance of an element and the dilatational wave speed still applies—although modest safety factors must be used in some instances.
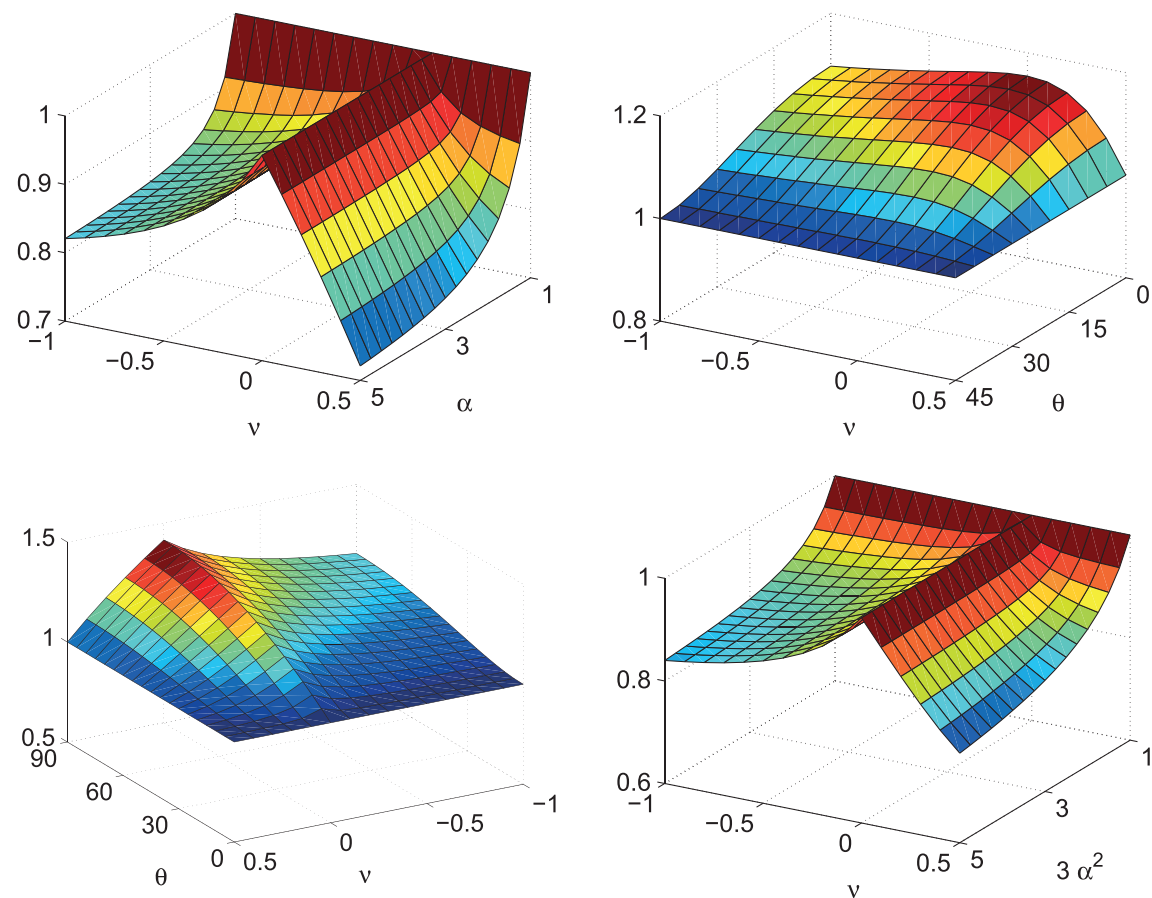

Figure 9. Maximum eigenfrequencies of distorted elements divided by those of undistorted elementsaspect ratio distortion of square elements (top left) and right-angled triangles (top right); angular distortion of square elements (bottom left) and isosceles triangles (bottom right). 


\section{CRITICAL TIME STEP FOR FINITE ELEMENT ASSEMBLIES}

Next, finite element assemblies of more than one element will be considered. As mentioned in the Introduction, most researchers estimate the critical time step by focussing on a single element, but according to Equation (1), the exact value of the critical time step is found by computing the eigenfrequencies of the entire finite element mesh, not of a single element in isolation -although the latter gives a safe bound to the exact (globally obtained) value of the critical time step [3].

Structured patches of multiple finite elements are generated by taking $n$ elements in each direction. For quadrilaterals, this means that $n \times n$ elements with identical dimensions are used. For triangles, each of the quadrilaterals is then subdivided into two triangles with identical dimensions (thus leading to $n \times n \times 2$ elements in total). As such, patches of distorted right-angled triangles and patches of distorted isosceles triangles are generated from initial grids of rectangles and parallelograms, respectively. Values for $n \in[1,2,4,8,16]$ are taken, and global stiffness and (lumped) mass matrices are generated using mass density $\rho=1 \mathrm{~kg} / \mathrm{m}^{3}$, Young's modulus $E=1 \mathrm{~N} / \mathrm{m}^{2}$ and Poisson's ratio $v=\frac{1}{4}$. The eigenfrequencies of the resulting system are then obtained in numerical (rather than symbolic) format and substituted into Equation (1) to obtain the critical time step.

In Figure 10, the critical time steps are plotted for the two types of elements and the two types of element distortion that are studied, using a range of element distortion parameters. It can be seen in all considered cases that larger patches of elements lead to larger values of the critical time step, although there seem to be asymptotic values for each combination of element type and distortion type. The main observations of Section 8 are also confirmed, namely that increased element distortion leads to increased critical time steps for rectangles and isosceles triangles, and to decreased critical time steps for right-angled triangles and rhombic elements. For rectangles (especially those with larger aspect ratios), the difference in critical time step between a single element $(n=1)$ and
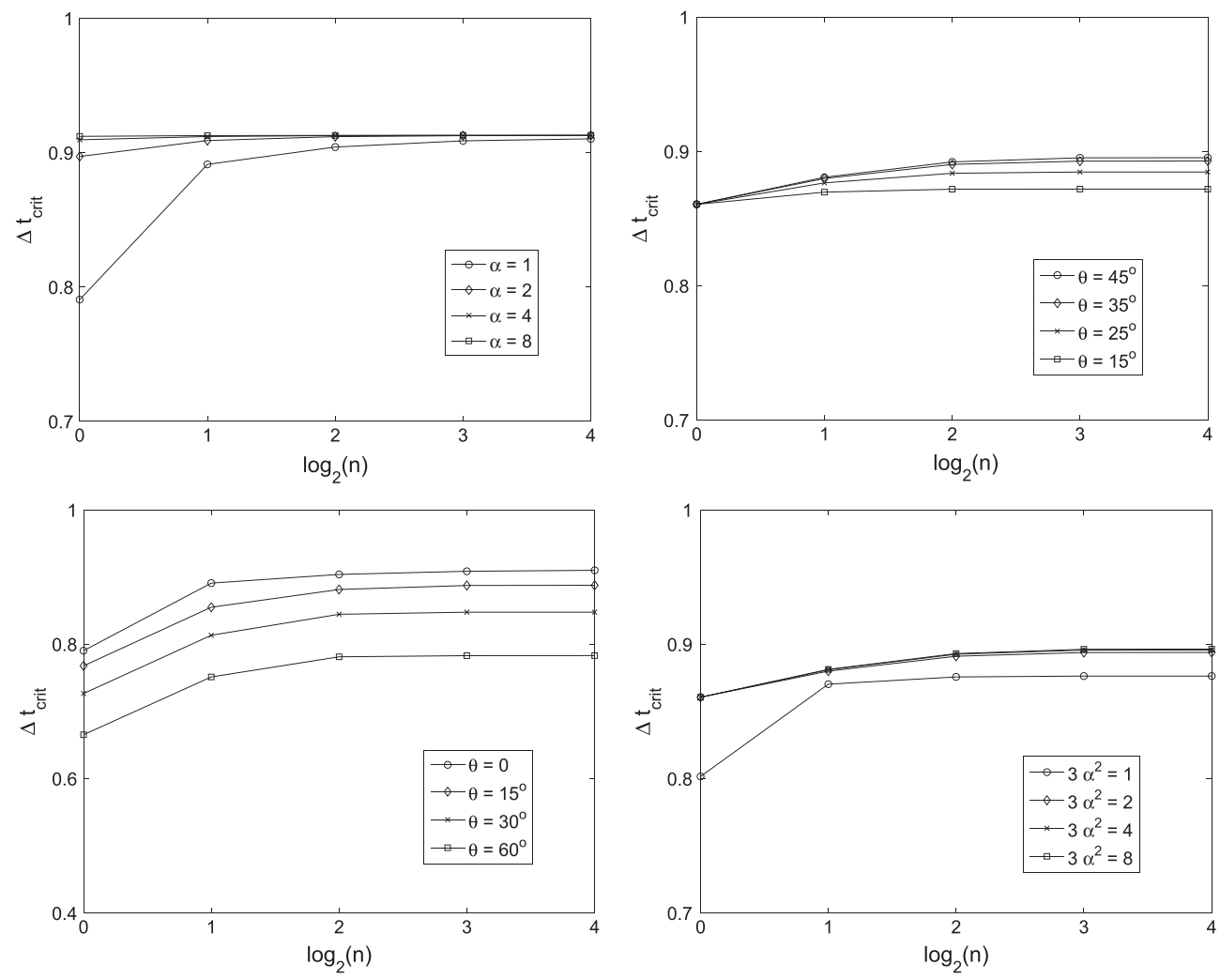

Figure 10. Critical time step $\Delta t_{\text {crit }}$ versus number of elements $n$-rectangular elements (top left), rightangled triangles (top right), rhombic elements (bottom left), and isosceles elements (bottom right). 
multiple elements is relatively small. On the other hand, there is up to $15 \%$ difference between the critical time step of a single rhombic element and multiple rhombic elements.

\section{CONCLUSIONS}

The effects of element distortion on the critical time step of low-order, two-dimensional finite elements have been studied in this paper, with focus on aspect ratio distortion and angular distortion of linear triangles and bilinear quadrilaterals. The dynamic eigenvalue problem has been formulated and solved on element level for a number of cases, and the maximum eigenfrequencies have been identified in symbolic form making use of appropriate parameters to capture the element distortion such as aspect ratio or change of internal angle. Thus, the maximum eigenfrequencies were found in terms of material parameters (dilatational wave speed and Poisson's ratio), the shortest distance within the element, and the element geometry.

The overriding conclusion is that element distortion can have an adverse effect on the critical time step; nevertheless, such effects tend to be limited. In many cases, there was in fact a slight beneficial effect of element distortion (that is, the critical time step was found to be increased by element distortion), whereas the most significant decrease of the critical time step was found to be a factor of $\sqrt{2}$. Thus, the rule-of-thumb used in practice that the critical time step equals the shortest distance divided by the dilatational wave speed, with a modest safety factor, can still be used.

An interesting secondary observation is that the integration rule for the spatial integration of the stiffness matrix does not affect the critical time step of quadrilateral elements. The reason is that the critical time step is set by the dilatational wave speed, whereas under-integration affects the shear wave speed but not the dilational wave speed. This holds for the undistorted square shape but also for distorted rectangular and rhombic shapes. The full derivations have not been presented in detail here, but they can be verified using the characteristic polynomials presented in Appendices A and $\mathrm{B}$, respectively.

\section{APPENDIX A: CHARACTERISTIC POLYNOMIALS FOR QUADRILATERAL ELEMENTS: SELECTIVE REDUCED INTEGRATION}

Selective reduced integration implies that the shear terms are under-integrated. This can be achieved by evaluating the third row of $\mathbf{B}$ as given in Equation (9) at the centre of the element only. For four-noded square elements, the eigenvalue problem of Equation (2) then reads

$$
\omega^{6} h^{6}\left(3 \omega^{2} h^{2}-4 c_{d}^{2}\right)^{2}\left(\omega^{2} h^{2}-8 c_{s}^{2}\right)^{2}\left(\omega^{2} h^{2}-8 c_{d}^{2}+8 c_{s}^{2}\right)=0
$$

The eigenvalue problem for a rectangular element with selective reduced integration can be written as

$$
\begin{gathered}
\omega^{6} h^{6}\left(3 \omega^{2} h^{2}-4 c_{d}^{2}\right)\left(3 \omega^{2} h^{2} \alpha^{2}-4 c_{d}^{2}\right)\left(\omega^{2} h^{2} \alpha^{2}-4 \alpha^{2} c_{s}^{2}-4 c_{s}^{2}\right) \\
\times\left(\omega^{4} h^{4} \alpha^{2}-4 \omega^{2} h^{2}\left(\alpha^{2}+1\right) c_{d}^{2}+64 c_{d}^{2} c_{s}^{2}-64 c_{s}^{4}\right)=0
\end{gathered}
$$

and for a rhombic quadrilateral element it reads

$$
\begin{gathered}
\omega^{6} h^{6}\left(9 \omega^{4} h^{4}-24 \omega^{2} h^{2} c_{d}^{2}+16 \sin ^{2}(2 \theta) c_{d}^{2} c_{s}^{2}-16 \sin ^{2}(2 \theta) c_{s}^{4}+16 \cos ^{2}(\theta) c_{d}^{4}\right) \\
\times\left(\omega^{2} h^{2}-8 c_{s}^{2}\right)\left(\omega^{4} h^{4}-8 \omega^{2} h^{2} c_{d}^{2}+64 \cos ^{2}(\theta) c_{d}^{2} c_{s}^{2}-64 \cos ^{2}(\theta) c_{s}^{4}\right)=0
\end{gathered}
$$

\section{APPENDIX B: CHARACTERISTIC POLYNOMIALS FOR QUADRILATERAL ELEMENTS: REDUCED INTEGRATION}

If reduced integration is applied to all three rows of matrix $\mathbf{B}$, the eigenvalue problem of Equation (2) for a four-noded square element reduces to

$$
\omega^{10} h^{10}\left(\omega^{2} h^{2}-8 c_{s}^{2}\right)^{2}\left(\omega^{2} h^{2}-8 c_{d}^{2}+8 c_{s}^{2}\right)=0
$$


Applying reduced integration to a rectangular element yields

$$
\omega^{10} h^{10}\left(\omega^{2} h^{2} \alpha^{2}-4 \alpha^{2} c_{s}^{2}-4 c_{s}^{2}\right)\left(\omega^{4} h^{4} \alpha^{2}-4 \omega^{2} h^{2}\left(\alpha^{2}+1\right) c_{d}^{2}+64 c_{d}^{2} c_{s}^{2}-64 c_{s}^{4}\right)=0
$$

and for a rhombic element this gives

$$
\omega^{10} h^{10}\left(\omega^{2} h^{2}-8 c_{s}^{2}\right)\left(\omega^{4} h^{4}-8 \omega^{2} h^{2} c_{d}^{2}+64 \cos ^{2}(\theta) c_{d}^{2} c_{s}^{2}-64 \cos ^{2}(\theta) c_{s}^{4}\right)=0
$$

As usual, the increased number of zero roots for $\omega$ corresponds to a number of unphysical zeroenergy modes.

\section{ACKNOWLEDGEMENT}

Financial support from the Royal Society under International Joint Project 'Bipenalty method for finite elements and explicit time integration' is gratefully acknowledged.

\section{REFERENCES}

1. Ling X, Cherukuri HP. Stability analysis of an explicit finite element scheme for plane wave motions in elastic solids. Computational Mechanics 2002; 29:430-440.

2. Hughes TJR. The Finite Element Method: Linear Static and Dynamic Finite Element Analysis. Prentice-Hall: Englewood Cliffs, New Jersey, 1987.

3. Lin JI. An element eigenvalue theorem and its application for stable time steps. Computer Methods in Applied Mechanics and Engineering 1989; 73:283-294.

4. Flanagan DP, Belytschko T. Eigenvalues and stable time steps for the uniform strain hexahedron and quadrilateral. ASME Journal of Applied Mechanics 1984; 51:35-40.

5. Belytschko T, Lin JI. Eigenvalues and stable time steps for the bilinear Mindlin plate element. International Journal for Numerical Methods in Engineering 1985; 21:1729-1745.

6. Lin JI. Bounds on eigenvalues of finite element systems. International Journal for Numerical Methods in Engineering $1991 ; 32: 957-967$.

7. Benson DJ. Stable time step estimation for multi-material Eulerian hydrocodes. Computer Methods in Applied Mechanics and Engineering 1998; 167:191-205.

8. Koteras JR, Lehoucq RB. Estimating the critical time-step in explicit dynamics using the Lanczos method. International Journal for Numerical Methods in Engineering 2007; 69:2780-2788.

9. Nakamachi E, Huo T. Dynamic-explicit elastic plastic finite-element simulation of hemispherical punch-drawing of sheet metal. Engineering Computations 1996; 13:327-338.

10. Chung WJ, Cho JW, Belytschko T. On the dynamic effects of explicit FEM in sheet metal forming analysis. Engineering Computations 1998; 15:750-776.

11. Kim J, Kang S-J, Kang B-S. A comparative study of implicit and explicit FEM for the wrinkling prediction in the hydroforming process. International Journal of Advanced Manufacturing Technology 2003; 22:547-552.

12. van den Boogaard AH, Huétink J. Simulation of aluminium sheet forming at elevated temperatures. Computer Methods in Applied Mechanics and Engineering 2006; 195:6691-6709.

13. Wang ZW, Zeng SQ, Yang XH, Cheng C. The key technology and realization of virtual ring rolling. Journal of Materials Processing Technology 2007; 182:374-381.

14. Aguinaga I, Fierz B, Spillmann J, Harders M. Filtering of high modal frequencies for stable real-time explicit integration of deformable objects using the finite element method. Progress in Biophysics and Molecular Biology 2010; 103:225-235.

15. Olovsson L, Unosson M, Simonsson K. Selective mass scaling for thin walled structures modeled with tri-linear solid elements. Computational Mechanics 2004; 34:134-136.

16. Hetherington J, Askes H. Penalty methods for time domain computational dynamics based on positive and negative inertia. Computers and Structures 2009; 87:1474-1482.

17. Askes H, Caramés-Saddler M, Rodríguez-Ferran A. Bipenalty method for time domain computational dynamics. Proceedings of the Royal Society A 2010; 466:1389-1408.

18. Hetherington J, Rodríguez-Ferran A, Askes H. A new bipenalty formulation for ensuring time step stability in time domain computational dynamics. International Journal for Numerical Methods in Engineering 2012; 90:269-286.

19. Hetherington J, Rodríguez-Ferran A, Askes H. The bipenalty method for arbitrary multipoint constraints. International Journal for Numerical Methods in Engineering 2013; 93:465-482.

20. Macek RW, Aubert BH. A mass penalty technique to control the critical time increment in explicit dynamic finiteelement analysis. Earthquake Engineering and Structural Dynamics 1995; 24:1315-1331.

21. Olovsson L, Simonsson K, Unosson M. Selective mass scaling for explicit finite element analyses. International Journal for Numerical Methods in Engineering 2005; 63:1436-1445. 


\section{H. ASKES, A. RODRÍGUEZ-FERRAN AND J. HETHERINGTON}

22. Plecháč $P$, Rousset M. Implicit mass-matrix penalization of Hamiltonian dynamics with application to exact sampling of stiff systems. Multiscale Modeling and Simulation (SIAM) 2010; 8:498-539.

23. Askes H, Nguyen DCD, Tyas A. Increasing the critical time step: micro-inertia, inertia penalties and mass scaling. Computational Mechanics 2011; 47:657-667.

24. Olovsson L, Simonsson K. Iterative solution technique in selective mass scaling. Communications in Numerical Methods in Engineering 2006; 22:77-82.

25. Lombardo M, Askes H. Lumped mass finite element implementation of continuum theories with micro-inertia. International Journal for Numerical Methods in Engineering 2013; 96:448-466.

26. Myers GE. The critical time step for finite-element solutions to two-dimensional heat-conduction transients. Journal of Heat Transfer 1978; 100:120-127. 